\title{
INTRACRANIAL SUPPURATION
}

\author{
Leslie Oliver, M.B.(Lond.), F.R.C.S., F.A.C.S. \\ Neurosurgeon, Charing Cross Hospital, West London Hospital and Royal Northern Hospital, London
}

Although the introduction of the sulphonamides and the antibiotics has reduced the incidence of intracranial suppuration, it can still cause considerable anxiety to the medical practitioner.

\section{Extradural Abscess}

An abscess may form between the skull and the dura mater as a complication of mastoiditis, osteomyelitis of the skull or fracture of the skull (open to the exterior, or into an infected mastoid process or nasal sinus). The dura mater forming the inner wall of the abscess is inflamed and usually covered with granulations corresponding to the extent of the abscess. There is severe toxæmia and pyrexia. Localizing neurological signs are sometimes found and depend on the site of the abscess. Rarely, osteomyelitis, bloodborne or arising from an overlying carbuncle, affects the suboccipital region causing an extradural abscess with cerebellar signs. The cerebrospinal fluid shows a moderate increase in white cells but contains no organisms; its pressure may be raised.

When an extradural abscess is caused by osteomyelitis or mastoiditis, the operation appropriate to these diseases establishes the necessary drainage. When, however, an extradural abscess follows fracture of the skull, a burr-hole is made over the site of the abscess and a drain inserted. Appropriate antibiotics are administered.

\section{Subdural Suppuration}

(The synonym purulent pachymeningitis is a bad term, for it equally well applies to extradural abscess which is associated with pachymeningitis secondary to overlying osteomyelitis.) Subdural empyema means a widespread collection of pus between the dura and arachnoid mater. It is an uncommon but serious condition which before the days of antibiotics was invariably fatal. The commonest cause is osteomyelitis of the skull following an acute exacerbation of inflammation of the nasal air sinuses. Such an exacerbation may be brought about by operative interference in sinusitis. Subdural infection may also be caused by blood-borne osteomyelitis of the skull, mastoiditis, or fractures of the skull which open to the exterior or into septic nasal sinuses of mastoid cells. Infected subdural effusions nøt infrequently complicate the convalescence in cases of acute purulent meningitis, especially in childe hood (Hankinson and Amador, 1956). There a\& severe toxæmia and pyrexia, with signs of raisea. intracranial pressure (headache, vomiting an papillodema). The infection spreads widely in the subdural space producing contralateral hemuparesis, cortical sensory loss and hemianopia. the dominant cerebral hemisphere is affected dysphasia or aphasia may be found. Patients sometimes present with grand mal or Jacksoniant epilepsy. Subdural empyemas sometimes occuip bilaterally.

A subdural abscess is a localized collection of pus; it may form during the treatment of sub dural empyema, or the infection may be localifed. from the beginning. Subdural abscesses tenct t\& form along the falx or between the occipital labe and tentorium. A subdural abscess produces localized neurological effect. Thus an abscess of the medial aspect of the hemisphere involving the motor cortex causes paralysis of the contras lateral leg with sparing of the arm and face.

Lumbar puncture in subdural infection shows raised pressure, and the cerebrospinal fluid con tains an increased number of white cells (100 to 300 per cu. mm.) but no organisms. Althoug a variety of organisms may be grown from the

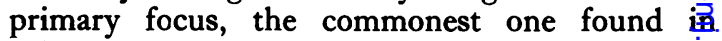
subdural pus is the non-hæmolytic streptococcus

Burr holes are made to confirm the diagnosts and to enable drainage to be carried out. If subdural empyema small rubber catheters are insinuated between the dura and arachnoid fof the instillation of penicillin, to which the organismis of subdural infection are usually sensitive (Schille Cairns and Russell, 1948). The strength of penicillin employed is 500 units per ml. of isotonic saline. Although systemic penicillin passes into the subdural space, the greater concentration of the drug obtained by the catheter method is desirable. Subdural abscesses are located with the aid of a brain cannula which is passed unhesitating through relatively silent parts of the brain, but it is particularly important to avoid the motor cortex 
and the speech zones. Diodone is injected into the subdural empyema or abscess cavity to demonstrate its extent radiologically. A subdural abscess, unlike a brain abscess, is shown to be superficial to the brain. Ventriculography or angiography may be required to localize it. Antibiotics are given from the beginning, and when penicillin is indicated, 20,000 units in $5 \mathrm{ml}$. of isotonic saline are given by lumbar puncture to prevent meningitis arising after needling the brain, for systemic penicillin does not reach the cerebrospinal fluid in sufficient concentration to be effective. Subdural abscess is treated by intermittent aspiration and usually the instillation of penicillin.

\section{Sigmoid Sinus Thrombosis}

Since the introduction of sulphonamides and antibiotics, the incidence of this condition has been greatly reduced and the clinical picture modified. Now it is more often a complication of chronic than of acute suppurative otitis media and, whereas in the past, septicæmia was the common presentation, today the manifestations of the disease are due to spreading thrombosis into other venous sinuses and the cerebral veins. Formerly, the common syndrome consisted of headache, rigors, swinging temperature, positive blood culture, and metastatic abscesses in the lungs and elsewhere; at the present time, the more usual features are headache, vomiting and papillodema caused by thrombosis spreading into the other venous sinuses; if it spreads into cortical veins, focal effects may be produced such as epilepsy or hemiparesis. If thrombosis spreads into the petrosal sinuses the fifth and sixth cranial nerves may be affected producing paralysis of the external rectus muscle and pain in the face (Gradenigo's syndrome). Extension of thrombosis into the internal jugular vein produces a tender swelling in the neck.

Opinion is divided about the extent of surgical treatment that is necessary in these days of abundant antibiotics. There is a tendency to be content with mastoidectomy and wide exposure of the sigmoid sinus, but some otologists still advocate the classical triad of mastoidectomy, evacuation of the clot from the sinus and ligation of the internal jugular vein (Reading and Schurr, 1956).

\section{Cavernous Sinus Thrombosis}

Septic thrombosis of the cavernous sinus may arise from: (a) Boils or carbuncles of the nose or upper lip, especially after surgical interference; (b) erysipelas; (c) dental sepsis in the upper jaw, especially after extractions; or $(d)$ middle-ear infection. In $(a),(b)$ and $(c)$ infection spreads to the sinus through the angular and ophthalmic veins; in $(d)$ it spreads through the superior petrosal sinus. The onset occurs with headache; rigors and a remittent temperature. The eyelids become swollen, the conjunctivæ œdematous, the eyes fixed and proptosed, and the pupils dilated and inactive. If the cornea is clear, retinal hæmorrhages and papillœdema may be observed.

Septic sinus thrombosis is rare in countries where sulphonamides and antibiotics are freely available for the treatment of the causal lesions. Formerly, the condition was fatal; patients died from septicæmia and metastatic infection of the lungs, or from meningitis; nowadays the disease is usually cured with antibiotics. Nevertheless; septic lesions of the nose or upper lip-the "danger area"-should not be squeezed or incised. There is nowadays no indication for ligation of the angular veins in cavernous sinus infection when the primary lesion is in the danger area.

\section{Meningitis}

Unless otherwise stated, the term meningitis refers to leptomeningitis (i.e. inflammation of the arachnoid and pia mater). Infection may reach the meninges directly from the exterior: $(a)$ by erosion of the skin over a cranial or spinal congenital defect (e.g. meningocele); $(b)$ as the result of open injuries of the head or spine; $(c)$ following cranial or spinal operations or lumbar puncture when aseptic technique has been defective. Infection may also reach the meninges from the following adjacent inflammatory diseases: (a) otitis media; (b) osteomyelitis of the skull; (c) infected dermoid sinus of the occiput or spine (Logue and Till, I952); (d) cerebral abscess, by rupturing into the ventricles or subarachnoid space. Lastly, infection may occur through the blood stream; tuberculous and meningococcal meningitis arise in this way whereas pneumococcal meningitis can occur either as a blood-borne infection from pneumonia, or directly from otitis media (by far the commonest cause) or nasal sinusitis. In many cases of pneumococcal meningitis the source of infection cannot be traced.

The symptoms of meningitis are frontal or occipital headache, vomiting and in some cases photophobia. Retention of urine may occur. The physical signs are neck stiffness, positive Kernig's sign and sometimes a positive Brudzinski's sign (flexion of the hips and knees occurs when the patient's head is flexed). These signs of meningeal irritation are accompanied by pyrexia and toxæmia. With the onset of coma, the signs of meningeal irritation may disappear, and in infants and young children they may be inconspicuous or absent from the beginning. In 
meningococcal meningitis, which may occur sporadically or in epidemics, a petechial rash may be present on the trunk ('spotted fever'). In tuberculous meningitis, the syndrome of meningeal irritation may be absent, and thus the ' diagnosis ' of pyrexia of unknown origin may be made.

In all forms of leptomeningitis, the cerebrospinal fluid pressure is raised. In pyogenic meningitis the fluid is opalescent because of the presence of a large number of polymorphs ( 1,000 to 10,000 per cu. mm.). The protein is increased ( 100 to $500 \mathrm{mg}$. per $100 \mathrm{ml}$.), while the sugar is reduced below $40 \mathrm{mg}$. per $100 \mathrm{ml}$. The chlorides are moderately reduced.

In tuberculous meningitis, the cerebrospinal fluid appears clear or slightly hazy and, after standing a few hours, a fine web-like fibrin clot forms. The cell count is increased. In the majority of cases there are between 50 and 500 cells per cu. mm. Lymphocytes and mononuclears predominate. The protein level is raised with an average of $200 \mathrm{mg}$. per $100 \mathrm{ml}$. If in tuberculous meningitis the protein exceeds 500 mg. per $100 \mathrm{ml}$. there is likely to be a subarachnoid block. The sugar content is lowered but not to the same extent as in pyogenic meningitis. The chloride is usually reduced below $65^{\circ} \mathrm{mg}$. per $100 \mathrm{ml}$. Although formerly thought to be a reliable test for the presence or absence of tuberculous meningitis, the chloride test is now regarded as significant only when positive. Under the microscope, tubercle bacilli may be seen in the fluid or can be cultured from it.

\section{Treatment of Meningitis}

In pyogenic meningitis, the organism should be identified as soon as possible and its antibiotic sensitivity determined. In the meantime, a wide spectrum antibiotic should be given. If the organism is sensitive to penicillin, the drug should be given intrathecally as well as intramuscularly, for an effective concentration of penicillin does not pass into the cerebrospinal fluid from the blood stream. The dose injected into the cerebrospinal fluid must not exceed 20,000 units daily and it should be dissolved in not less than $5 \mathrm{ml}$. of isotonic saline. Cases are on record in which 500,000 units were given in error and the patients developed generalized convulsions and died within 24 hours. Pneumococcal meningitis is usually treated with penicillin and a sulphonamide, whereas meningococcal meningitis requires only a sulphonamide in the majority of cases. Hamofhilus influenza meningitis usually responds to chloramphenicol alone, although there is a tendency to give it with sulphadiazine. In pyogenic meningitis, the focus of origin of the infection may be known and can be treated surgically, e.g. mastoiditis and osteomyelitis of the skull. Iñ tuberculous meningitis, a course of streptomycin is given lasting several months. The drug is giver intramuscularly and intrathecally. Burr holes. may be needed for injection of the drug into the lateral ventricles when adhesions have produce a spinal block (the intrathecal or intraventriculas dose of streptomycin is 50 to $100 \mathrm{mg}$. in $5 \mathrm{ml} \frac{\mathrm{O}}{\mathrm{N}}$ isotonic saline). Burr holes may also be requirect in pneumococcal meningitis when a spinal block occurs. When satisfactory progress is not being made by a patient suffering from pyogeni $\vec{b}$ meningitis, the possibility of a coexistent brain abscess, subdural abscess or subdural hygroms should be considered.

\section{Brain Abscess}

Otitis media and mastoiditis may give rise tơ an abscess in either the temporal lobe or the cerebellum. Abscess of the brain is much mor $\vec{\xi}$ likely to be caused by chronic than acute infection of the middle ear. Inflammatory diseases of the lungs, especially following their surgical treatment, are liable to cause metastatic brain abscesses $\$$ single abscesses occur much more frequently thares multiple ones. Degeneration of a secondar carcinoma, especially when there is no evide primary growth, may cause confusion in diagnogis when the lesion in the brain is aspirated. Nagade sinusitis, with or without spreading osteomyelieis? may cause a frontal lobe abscess. Open fractu站官 of the skull, when the dura mater has been opene电 and the brain penetrated, have a strong tendency to cause intracranial suppuration (Fig. I). brain abscess is almost certain to form if frag ments of bone, hair or hat material have been left in the brain. Fractures into the nasal aiê sinuses, with or without cerebrospinal fluid rhinorrhœa, frequently cause meningitis and some times a cerebral abscess. The presence of missile is much less likely to cause an abscess Osteomyelitis of the skull, caused either by. infection of the nasal air sinuses or by blood-borne. organisms, is liable to cause a brain abscessi Occasionally an inflammatory lesion of the scaly produces a cerebral abscess without evidence of osteomyelitis. Rarely, infection of an operation wound causes an abscess of the brain. Periphera sepsis is responsible for an occasional brai abscess. Examples of such causal lesions arer carbuncle, whitlow, topsillitis, infected dental root (especially after extraction), septic wounds and osteomyelitis. Sometimes an abscess occurg without an evident focus of infection and mas thus be encountered unexpectedly. Cryptogenic brain abscess is particularly liable to occur in th presence of congenital heart disease, especially when there is cyanosis (Campbell, 1957). More 


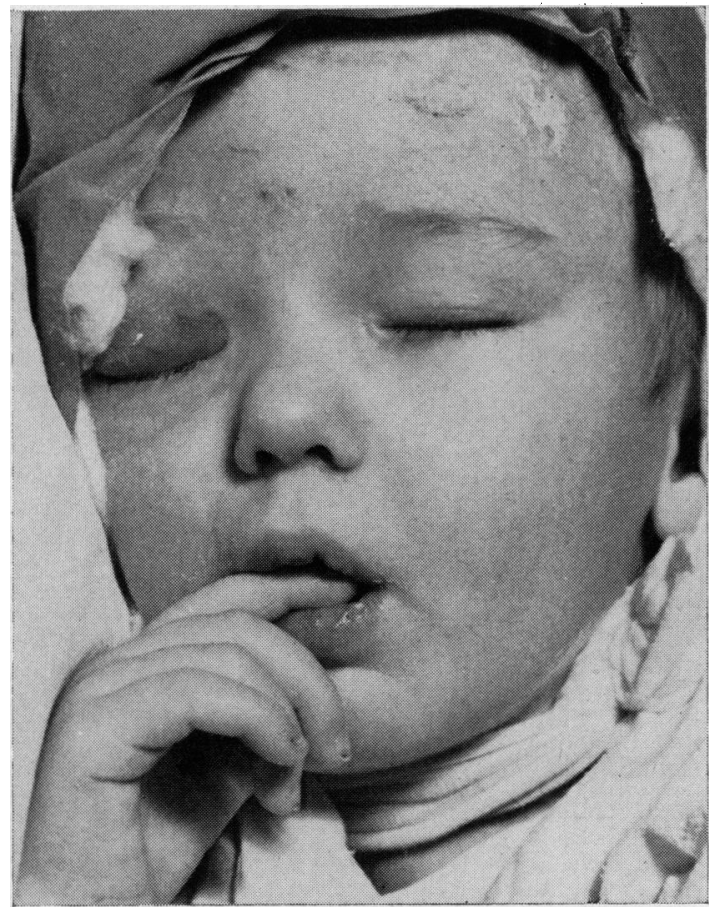

FIG. I.- Inflammation of the right upper lid at the site of entry of a pencil through the root of the orbit. The pencil entered the brain and caused an abscess in the frontal lobe. The abscess was successfully treated by the aspiration method.

than $50 \%$ of such abscesses have been found in association with Fallot's tetralogy and some have followed operations for cardiac lesions. They are not necessarily caused by paradoxical emboli, for the shunt is not always from right to left.

Bacteriology. Organisms isolated from abscesses of the brain include Staphylococcus aureus or albus; Streptococcus pyogenes, viridans or pneumonia Pseudonomas pyocyanea; Proteus vulgaris; actinomyces; diphtheroids; Hamophilus influenza and occasionally Entameba histolytica. Not infrequently a mixed growth of organisms is obtained. The nature of the responsible organisms and their sensitivity to the various antibiotics is ascertained. Often, however, no organism is demonstrated in the pus although the infection may still be active in the abscess capsule.

Diagnosis. An abscess of the brain behaves like a tumour, giving rise to increased intracranial pressure and localizing signs. Thus there are headache, vomiting and, frequently, but not always, a mild degree of papillœdema. A metastatic abscess may begin with epileptic attacks which, if of the Jacksonian type, are of localizing value. When the infection arises in the skull or its air-containing cavities, the cerebral abscess is

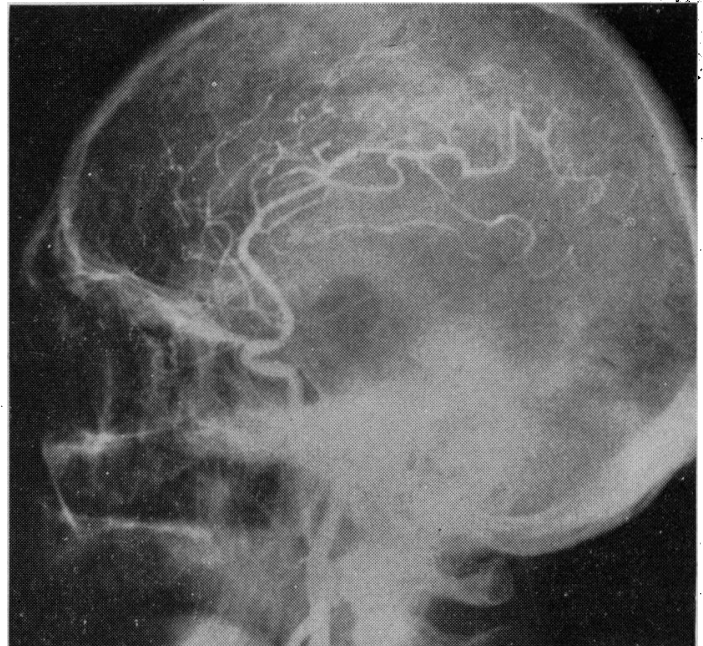

FIG. 2.-A carotid arteriogram showing the characteristic upward sweep of the middle cerebral artery and the opening up of the carotid 'syphon' produced by a space-occupying lesion in the temporal lobe, in this case an abscess.

usually immediately subjacent to the primary lesion, but occasionally it may be well away from the area of osteomyelitis. A careful neurological examination is therefore necessary. The commonest site for osteomyelitis is in the frontal region, although no part of the skull is exempt. Of particular importance in temporal lobe abscesses is examination of the visual fields which commonly show an upper quadrantic homonymous defect or, if the abscess is extensive, a complete hemianopia. Cerebellar abscesses produce minimal or moderate cerebellar signs (unilateral hypotonia; incoordination of the upper extremity of the same side; nystagmus, which is more marked and of greater excursion when the patient fixes his gaze towards the side of the abscess; and a tendency for the patient to deviate towards the side of the lesion when walking). A metastatic abscess may occur anywhere in the brain. As much information as possible about its location is obtained from the history and neurological examination, as in the diagnosis of intracranial tumours, and help then obtained from the investigations discussed below. The temperature is most likely to be slightly raised $\left(99^{\circ}\right.$ to $100^{\circ} \mathrm{F}$.) or subnormal. A high temperature is not found with an uncomplicated brain abscess. The pulse may show an increased rate or the classical slowing of increased intracranial pressure.

Investigations. Lumbar puncture is avoided when possible for fear of producing a cerebellar pressure cone. The cerebrospinal fluid is clear in uncomplicated cases, although there is usually 
Fig. 3.-A temporal lobe abscessc visualized radiologically by in $-\Rightarrow$ jection of $2 \mathrm{ml}$. diodone after aspiration of pus.

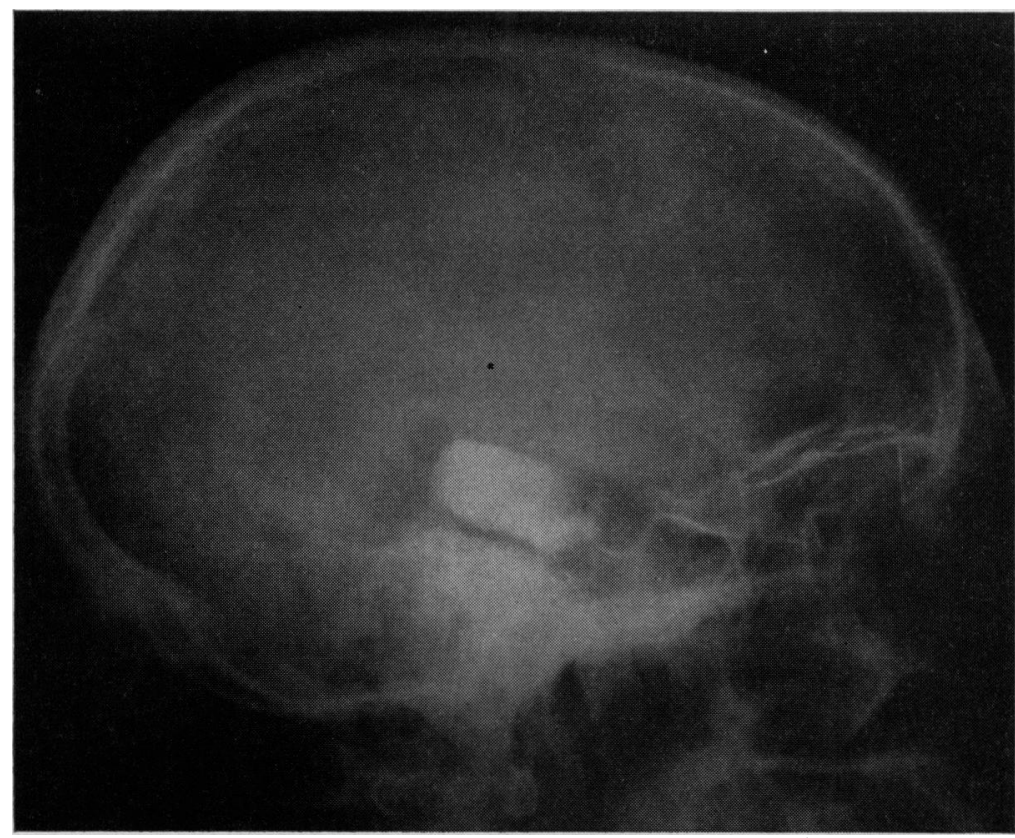

a closed abscess cavity is asking for persistence 하믐

a moderate increase in white cells. There should be no organisms, either on direct examination of the fluid or after culture. When there is doubt about the presence or location of an abscess, ventriculography or arteriography should be carried out. The latter investigation is particularly useful in demonstrating an abscess in the temporal lobe, because it may be difficult or impossible to fill the temporal horn with air in such cases (Fig. 2). Electroencephalography is likely to show slow waves of marked amplitude, and phasereversal in the region of a supratentorial abscess. When a brain abscess has been localized, its extent can be demonstrated by positive contrast radiography. Diodone 2 to $3 \mathrm{ml}$. is injected into the abscess cavity after some of the pus has been aspirated (Oliver and Leese, 1949). The skull is then X-rayed (Fig. 3). Many neurosurgeons recommend Thorotrast (thorium dioxide), for it has the advantage of remaining in situ and thus the progress of the abscess can be followed radiologically. But there is experimental evidence that Thorotrast, acting as a foreign body, stimulates capsule formation (Falconer, McFarlan and Russell, I943). Therefore, if resolution of the abscess is desired, the rapidly absorbable contrast medium, diodone, should be employed. Furthermore, it is a violation of surgical principles to leave an unabsorbable foreign body in a septic place. The late Julian Taylor had strong feelings about this: 'I should like to point out that to inject a foreign body such as Thorotrast ... into infection as plainly in the brain as it would be anywhere else in the body. There is no primaxy reason to suppose that it may be any less provoco tive of chronicity than is a sequestrum, or a knat of so-called absorbable catgut, in the depths of a sinus. I cannot help thinking that the widespread habit of demonstrating the existence of abscesses radiclogically by this means may bee a cause of the need to excise them later' (Taylor, 1950).

\section{Treatment of Brain Abscess}

(a) Aspiration. A burr hole is made over the site of the abscess, unless it is secondary to osteomyelitis of the skull when the dura mater is:already exposed by removal of infected bone 3 . A small incision, a few millimetres long, is mado in the dura mater. An avascular point on the surface of the brain is punctured with a pointed tenotome, and a blunt-ended brain needle is passed into the abscess cavity. The pus is aspirated and replaced by 2 to $3 \mathrm{ml}$. penicillin solution (I00,000 units per ml.). This smalks volume of fluid is injected to avoid rupture of the capsule which may be extremely thin. Sometimeș an abscess heals after one penicillin replacement ${ }_{\sigma}^{\omega}$ but the procedure may have to be repeated severak times. The cannula (brain needle) is then passec through the original burr hole between thes sutures. Considerable judgment is needed in spacing aspirations. Needling should be repeated 


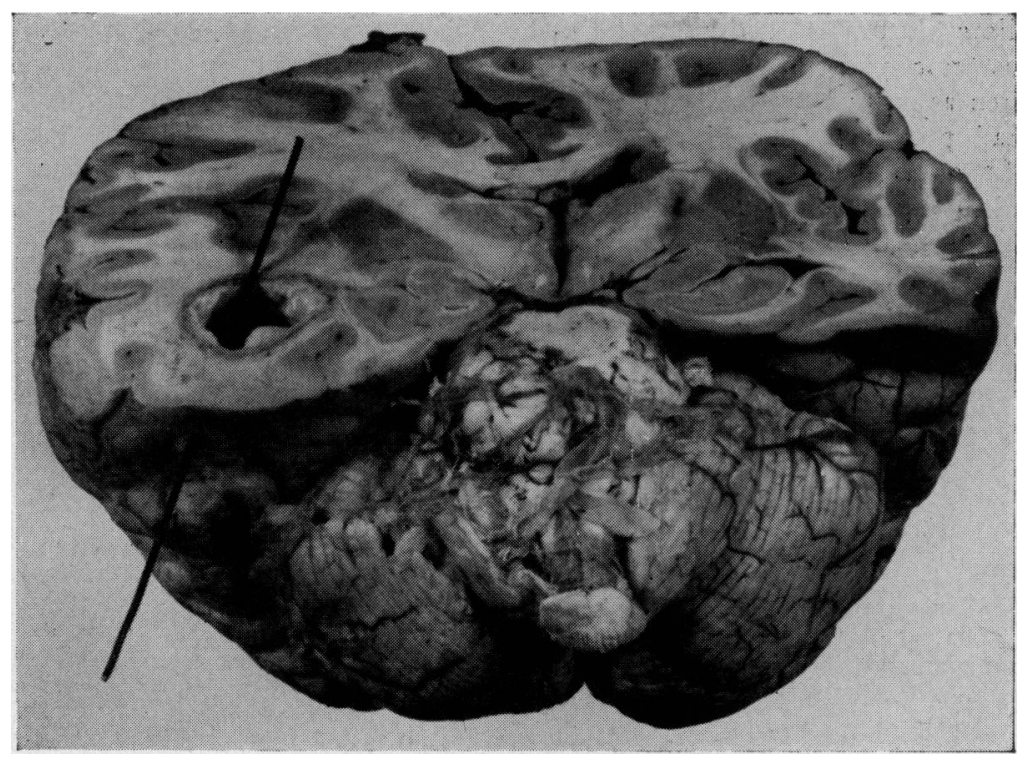

Fig. 4.-The probe indicates a large opening from the interior wall of a temporal abscess through which there was a free communication with the tympanic antrum. This type of abscess should be totally excised.

if the patient's general and neurological state do not improve; it should not be delayed until deterioration occurs. At first, aspiration and penicillin replacement may be needed at intervals of 24 to 48 hours, but when progress is satisfactory, the intervals become longer and longer until no more pus can be aspirated. Many abscesses can be completely cured by this technique (Oliver and Leese, 1949) but if a patient's condition does not improve or deteriorates the possible causes are: (I) Persistent infection in the capsule associated with brain swelling. The abscess should then be excised (vide infra, method $b$ ) and decompression performed. The odema may also be reduced by intravenous injection of a solution of urea. (2) Loculation, in which aspiration only drains part of the abscess. Other loculi may be detected and drained by needling in new directions. (3) The presence of multiple abscesses which may occur when the primary infection is in the lungs. Neurological examination alone may not reveal the location of additional abscesses, but they may be demonstrated by ventriculography. (4) A sinus which has become established with the tympanic antrum in an otogenic brain abscess (Fig. 4). Such an abscess will not heal unless excised. (5) The presence of a foreign body in the abscess, e.g. bone fragments in posttraumatic cases. The aspiration technique is particularly applicable to abscesses which involve the motor cortex, the speech zones or the optic radiations when other methods would cause severe neurological deficits. Furthermore, aspiration may be life-saving for patients who are too ill to undergo a major operation.

(b) Secondary Excision. Excision of the abscess capsule is carried out when aspiration alone fails. Some neurosurgeons, however, excise the abscess capsule as a routine as soon as aspiration is unproductive. After excision of the abscess capsule, the wound is closed without drainage.

(c) Primary Excision. This is another method of treatment made possible by the discovery of antibiotics (Le Beau, 1946). The advantage is that the anxious period of observation required by the aspiration technique is eliminated. Primary excision of the abscess is particularly indicated when it is situated in a relatively silent part of the brain, e.g. the frontal or cerebellar lobes where a neurological deficit is unlikely to be caused by excision. It is also the best method of treating an abscess following an open head injury, for any indriven fragments of bone or foreign bodies are automatically removed and the infection is thus brought to an end. A brain abscess occasionally follows a very slow course, and the onset of increased intracranial pressure and localizing signs are long delayed. Such an abscess develops into a solid mass of fibrous tissue closely resembling a tumour, although there may be a small quantity of pus in the centre. Primary excision is the only possible treatment in such a case.

In the management of cerebellar abscess, compression of the medulla oblongata by the increasing volume of the abscess and the surrounding œdema 
of the cerebellum demands immediate decompression. A full exposure of the posterior fossa is first carried out. The abscess is then aspirated with a brain cannula through a small puncture made in the dura mater on the side of the abscess, and penicillin (100,000 units dissolved in $\mathrm{I} \mathrm{ml}$. of isotonic saline) is instilled into the cavity. The dura mater is opened over both cerebellar lobes, and the abscess is removed. The wound is closed without drainage, and 20,000 units of $\stackrel{\Phi}{\circ}$

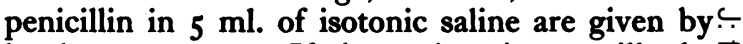
lumbar puncture. If the patient is very ill, the abscess is aspirated and its removal carried outa few days later.

\section{REFERENCES}

Campbell, M. (1957): Cerebral Abscess in Cyanotic Congenital Heart Disease, Lancet, i, 1 I 1 .

Falconer, M. A., McFarlan, A. M., Russell, D. S. (1943): Experimental Brain Abscesses in the Rabbit, Brit. $\Im . \circlearrowleft$ Surg., 30, 245.

Hankinson, J., and Amador, L. V. (1956): Infected Subdural Effusions, Brit. med. F. ii, 122.

Le BeAu, J. (1946): Radical Surgery and Penicillin in Brain Abscess, $\mathcal{F}$. Neurosurg., $3,359$.

Logue, V., and Till, K. (1952): Posterior Fossa Dermoid Cysts with Special Reference to Intracranial Infection, Э. Neurol. Neurosurg. Psychiat., 15, 1 .

Oliver, L. C., and LeEse, W. L. B. (1949): Treatment of Brain Abscess with Special Reference to a Conservative Method, Lancet, ii, 828.

Reading, P. V., and SchurR, P. H. (1956): Thrombosis of the Sigmoid Sinus, Ibid., ii, 473.

Schiller, F., Cairns, H., and Russell, D. S. (1948): The Treatment of Purulent Pachymeningitis and Subdural Suppuration with Special Reference to Penicillin, f. Neurol. Neurosurg. Psychiat., II, New Series, 143.

TAylor, J. (1950): Intracranial Suppuration, Proc. roy. Soc. Med., 43, 129. 